\title{
Surgical Treatment of Carpal Tunnel Syndrome through a Minimal Incision on the Distal Wrist Crease: An Anatomical and Clinical Study
}

\author{
Hye Mi Yoo, Kyoung Suk Lee, Jun Sik Kim, Nam Gyun Kim \\ Department of Plastic and Reconstructive Surgery, Institute of Health Sciences, GNUH Gyeongsang National University School of Medicine, \\ Jinju, Korea
}

Background An anatomical analysis of the transverse carpal ligament (TCL) and the surrounding structures might help in identifying effective measures to minimize complications. Here, we present a surgical technique based on an anatomical study that was successfully applied in clinical settings.

Methods Using 13 hands from 8 formalin-fixed cadavers, we measured the TCL length and thickness, correlation between the distal wrist crease and the proximal end of the TCL, and distance between the distal end of the TCL and the palmar arch; the TCL cross sections and the thickest parts were also examined. Clinically, fasciotomy was performed on the relevant parts of 15 hands from 13 patients by making a minimally invasive incision on the distal wrist crease. Postoperatively, a two-point discrimination check was conducted in which the sensations of the first, second, and third fingertips and the palmar cutaneous branch injuries were monitored (average duration, 7 months).

Results In the 13 cadaveric hands, the distal wrist crease and the proximal end of the TCL were placed in the same location. The average length of the TCL and the distance from the distal TCL to the superficial palmar arch were $35.30 \pm 2.59 \mathrm{~mm}$ and $9.50 \pm 2.13 \mathrm{~mm}$, respectively. The thickest part of the TCL was a region $25 \mathrm{~mm}$ distal to the distal wrist crease (average thickness, $4.00 \pm 0.57 \mathrm{~mm}$ ). The 13 surgeries performed in the clinical settings yielded satisfactory results.

Conclusions This peri-TCL anatomical study confirmed the safety of fasciotomy with a minimally invasive incision of the distal wrist crease. The clinical application of the technique indicated that the minimally invasive incision of the distal wrist crease was efficacious in the treatment of the carpal tunnel syndrome.

Keywords Carpal tunnel syndrome / Median nerve / Anatomy / Wrist / Cadaver
Correspondence: Nam Gyun Kim Department of Plastic and Reconstructive Surgery, Institute of Health Sciences, GNUH Gyeongsang National University School of Medicine, 79 Gangnam-ro, Jinju 660-702, Korea

Tel: $+82-55-750-8129$

Fax: +82-55-758-6240

E-mail: pellow@hanmail.net

Received: 12 Dec 2014 • Revised: 29 Jan $2015 \bullet$ Accepted: 25 Feb 2015

pISSN: 2234-6163 • elSSN: 2234-6171 • http://dx.doi.org/10.5999/aps.2015.42.3.327• Arch Plast Surg 2015;42:327-333

This article was presented as a poster at the 73th Congress of Korean Society of Plastic and Reconstructive Surgeons on November 7-9, 2014 in Seoul, Korea

No potential conflict of interest relevant to this article was reported.

\section{INTRODUCTION}

Carpal tunnel syndrome (CTS) was introduced in 1854 when James Paget first described a case of median nerve compression at the wrist in a distal radius fracture $[1,2]$. Since James Lear- month performed the first open release of the transverse carpal ligament (TCL) in 1933, CTS has been the most frequently prevalent entrapment neuropathy $[2,3]$. The latest data estimate the prevalence of CTS at 50 cases per 1,000 persons, with its incidence estimated at $1-3$ per 1,000 persons. Open carpal tunnel 
release surgery has been the most frequently performed surgery on the hand over the past 2 decades [4]. The following are the six diagnostic criteria for CTS: (1) nocturnal numbness, (2) numbness and tingling in the median nerve distribution, (3) weakness and/or atrophy of the thenar muscles, (4) Tinel's sign, (5) Phalen's sign, and (6) loss of two-point discrimination [5]. For early CTS, non-surgical treatment, such as a splint or local steroid injection, is recommended. However, surgery is recommended in cases of rapidly progressing thenar muscle atrophy and hand dysfunction, persisting severe symptoms after conservative treatment for 2-7 weeks, persistent symptoms or signs of disability evidenced by electromyography, or aggravated twopoint discrimination [6].

To date, a variety of surgical methods, such as open, limited incision, and endoscopic techniques for the treatment of CTS have been described, and their advantages and disadvantages have been discussed in a number of publications. Such surgical methods generally show a high success rate and low postoperative morbidity; however, unexpected poor outcomes can arise from an insufficient understanding of anatomical and pathophysiological characteristics of this disease and insufficient knowledge of diagnostic and therapeutic details by the surgeon. Consequently, it is important to develop a safe and efficacious surgical method that can minimize incision-induced scars and avoid intraoperative complications such as injuries to the palmar cutaneous branch of the median nerve, thenar branch, or median, ulnar, and digital nerves as well as damage to the superficial palmar arch, thus enabling an early return to preoperative activities. Against this background, we conducted an anatomical study to investigate the precise locations of the peri-TCL structures; then, we successfully performed TCL release with a minimally invasive incision to the distal wrist crease by thoroughly applying the findings of the anatomical study in clinical settings, without any intraoperative complications.

\section{METHODS}

\section{Anatomical study}

We dissected 13 intact hands preserved on 8 formalin-fixed cadavers that were placed in the supine position, while wearing surgical magnifiers. Of the 8 cadavers, 6 were men and 2 were women. 7 patients (87.5\%) were over 60 years of age, and 1 patient $(12.5 \%)$ was under 60 years. The incision line was defined by marking the intersection between the palmar Kaplan's cardinal line and a longitudinal line from the third web space to the distal wrist crease along the third digit. An incision line was drawn on the distal wrist crease. We located the TCL as well as the proximal peri-TCL regions after bilaterally opening the skin flaps from the marked intersection. After opening the palmar fat pads horizontally, we resected the palmar fascia and interthenar fascia and moved them to the side to locate the thick TCL and the superficial palmar arch distal to the TCL. Then, we measured the length and the thickness of each component and investigated the proximity between individual structural elements.

\section{Clinical study}

Between May 2013 and September 2014, we diagnosed CTS in patients (average age, 55.3 years) with Tinel's sign [7], Phalen's sign [8], and continuous numbness and tingling sensation in the fingers [9], and performed TCL fasciotomy with a minimally invasive incision of the distal wrist crease $(1.5-2 \mathrm{~cm})$ in 15 hands from 13 patients for whom surgery was indicated. The patients were monitored for 14 months; the most frequent underlying diseases were diabetes, hypertension, and renal failure. Two-point discrimination tests were performed to determine the objective preoperative and postoperative functions of the subjects, and the subjective symptoms and functions were determined using a questionnaire. If a patient recovered $100 \%$ of the baseline function of the unaffected hand (such as gripping a ballpoint pen, buttoning one's own shirt, holding and viewing a book, holding a telephone receiver, and mopping), the individual's performance was marked as "excellent"; $50 \%$ or more, as "good"; and no recovery, as "bad." The pre- to postoperative change in sensation was determined using the two-point discrimination test with a caliper on the thenar and hypothenar areas of the affected side, and the postoperative damage to the palmar cutaneous branch of the ulnar and radial nerves. The average two-point discrimination value on the unaffected hand was approximately $10 \mathrm{~mm}$.

\section{Surgical study}

With the patient in the supine position, surgery was performed under local anesthesia without using a tourniquet. We drew a $1.5-2.0-\mathrm{cm}$ transverse incision line on the distal wrist crease within the window formed by the palmaris longus tendon, the flexor carpi ulnaris tendon, Kaplan's cardinal line, and a longitudinal line from the third web space on the palm (Fig. 1A). Local anesthesia was administered along the line on the palmar side stretching from the distal wrist crease to Kaplan's line as well as along the incision line on the distal wrist crease. After sufficient anesthesia was administered, the distal wrist crease was dissected, and the sites of bleeding were electrocauterized. The incision was extended over the palmaris longus tendon, and care was taken to avoid damage to the cutaneous branch while performing the resection. After the skin incision, we located the thick TCL glowing after obtaining a free view by pushing the fat 


\section{Fig. 1. Operative photograph of a patient}

(A) A 1.5-cm incision line was drawn on the distal wrist cease, and the intersection of the longitudinal line from the third web of the third digit proximal to the ulnar side and Kaplan's cardinal line was marked. After drawing a right angle from this intersection and the incision line, the regions $25 \mathrm{~mm}$ and $35 \mathrm{~mm}$ distal to the incision line were marked. (B) The transverse carpal ligament was clearly exposed to view by pulling the fat back with the retractor and horizontally widening the gap after a transverse incision on the distal wrist crease; a longitudinal incision to the ulnar aspect was performed. (C) Primary closure of the 2-cm incision line of the distal wrist crease.
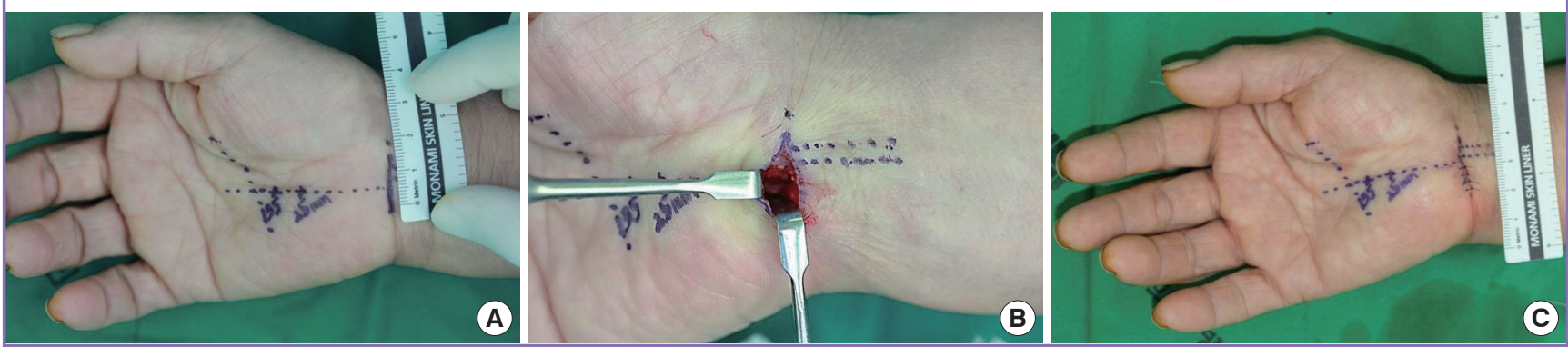

Table 1. Cadaver dissection; measurement of the TCL and the adjacent structures

\begin{tabular}{|c|c|c|c|c|c|c|c|}
\hline No. & Age/Sex & Right/Left & $\begin{array}{l}\text { Height } \\
(\mathrm{cm})\end{array}$ & $\begin{array}{l}\text { TCL length } \\
(\mathrm{mm})\end{array}$ & $\begin{array}{l}\text { TCL thickness } \\
(\mathrm{mm})\end{array}$ & $\begin{array}{l}\text { TCL-SPA } \\
(\mathrm{mm})\end{array}$ & $\begin{array}{l}\text { Thickness part-DWC } \\
\qquad(\mathrm{mm})\end{array}$ \\
\hline 1 & 70/Male & $\begin{array}{l}\text { Right } \\
\text { Left }\end{array}$ & 160 & 35 & 3 & $\begin{array}{c}10.8 \\
-\end{array}$ & 24 \\
\hline 2 & 65/Female & $\begin{array}{l}\text { Right } \\
\text { Left }\end{array}$ & 165 & $\begin{array}{l}34 \\
-\end{array}$ & $\begin{array}{l}4 \\
-\end{array}$ & 10 & 25 \\
\hline 3 & 50/Female & $\begin{array}{l}\text { Right } \\
\text { Left }\end{array}$ & 160 & $\begin{array}{l}33 \\
35\end{array}$ & $\begin{array}{l}4 \\
4\end{array}$ & $\begin{array}{l}10.5 \\
10\end{array}$ & $\begin{array}{l}25 \\
24\end{array}$ \\
\hline 4 & 88/Male & $\begin{array}{l}\text { Right } \\
\text { Left }\end{array}$ & 174 & $\begin{array}{l}38 \\
38\end{array}$ & $\begin{array}{l}4 \\
4\end{array}$ & $\begin{array}{r}9.5 \\
10.8\end{array}$ & $\begin{array}{l}26 \\
26\end{array}$ \\
\hline 5 & 67/Male & $\begin{array}{l}\text { Right } \\
\text { Left }\end{array}$ & 172 & $\begin{array}{l}37 \\
33\end{array}$ & $\begin{array}{l}5 \\
5\end{array}$ & $\begin{array}{r}9.9 \\
10.3\end{array}$ & $\begin{array}{l}25 \\
25\end{array}$ \\
\hline 6 & 68/Female & $\begin{array}{l}\text { Right } \\
\text { Left }\end{array}$ & 179 & $\begin{array}{l}38 \\
40\end{array}$ & $\begin{array}{l}4 \\
4\end{array}$ & $\begin{array}{l}10 \\
10.4\end{array}$ & $\begin{array}{l}25 \\
26\end{array}$ \\
\hline 7 & 60/Female & $\begin{array}{l}\text { Right } \\
\text { Left }\end{array}$ & 149 & $\begin{array}{l}32 \\
33\end{array}$ & $\begin{array}{l}4 \\
3\end{array}$ & $\begin{array}{l}9.8 \\
2.6\end{array}$ & $\begin{array}{l}25 \\
25\end{array}$ \\
\hline 8 & 77/Female & $\begin{array}{l}\text { Right } \\
\text { Left }\end{array}$ & 158 & 33 & $\begin{array}{l}4 \\
-\end{array}$ & 8.9 & $\begin{array}{c}24 \\
-\end{array}$ \\
\hline Mean \pm SD & - & - & - & $35.30 \pm 2.59$ & $4.00 \pm 0.57$ & $9.50 \pm 2.13$ & $25.00 \pm 0.71$ \\
\hline
\end{tabular}

pad laterally to the retractor while vertically dissecting using Metzenbaum scissors. After procuring a free view by distally dissecting the TCL with a free elevator, longitudinal resection of the proximal TCL was conducted with a \#15 blade, and sufficient dissection of the TCL was confirmed by checking against the median nerve, which is surrounded by the synovium and the tendon. Using a pair of blunt-ended face-lift scissors, we slowly conducted resection distal to the TCL; maximum distance was achieved proximal to the ulnar side of the third web. Resection continued until the region $35 \mathrm{~mm}$ distal to the distal wrist crease and the region at the thickest section ( $25 \mathrm{~mm}$ away) were sufficiently resected. Finally, to ensure that the TCL was completely dissected, we determined that there was no resistance by shaking the tip of the Metzenbaum scissors vertically over the TCL (Fig. 1B). After sufficient irrigation along the incision site and confirmation of no other bleeding sites, the residual TCL on the wrist side was sufficiently dissected to completely release compression, followed by interrupted suture of the skin with blue nylon 5-0; placement of a soft, bulky dressing with mild compression; and application of a splint in a mild wrist extension position (Fig. 1C).

\section{Statistical analysis}

The statistical analysis was performed using IBM SPSS ver. 20.0 (IBM Co., Armonk, NY, USA). The means \pm standard deviations of the quantitative variables were calculated. Values are presented as number (in millimeters).

\section{RESULTS}

\section{Anatomical study}

Table 1 shows the measurement of the TCL and the adjacent 


\section{Fig. 2. Cadaver dissection of the hand}

The length from the distal TCL to the SPA and the length and thickness of the TCL are measured with a millimeter-unit ruler. The locations of the DWC and the proximal carpal tunnel ligament are almost identical. TCL, transverse carpal ligament; SPA, superficial palmar arch; DWC, distal wrist crease.

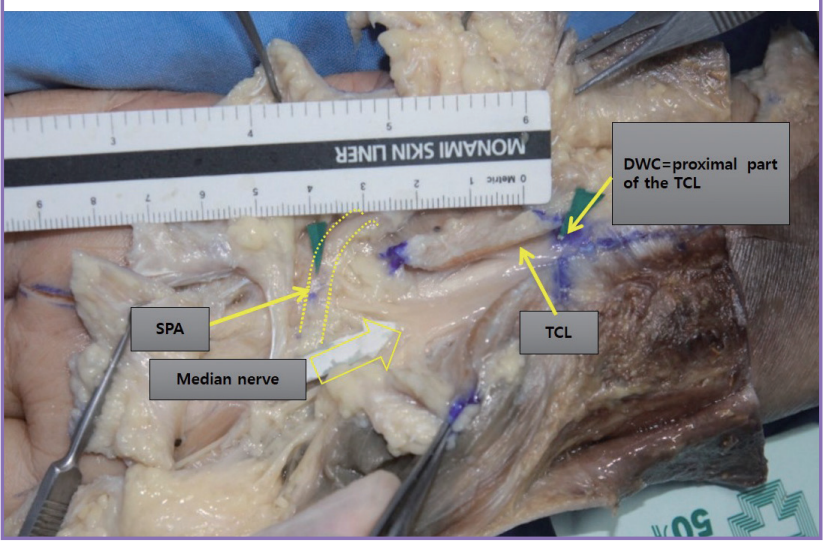

Fig. 3. Cross section of the TCL

Cross section of the TCL (arrow). The region of the TCL $25 \mathrm{~mm}$ distal to the distal wrist crease was the thickest part. TCL, transverse carpal ligament.

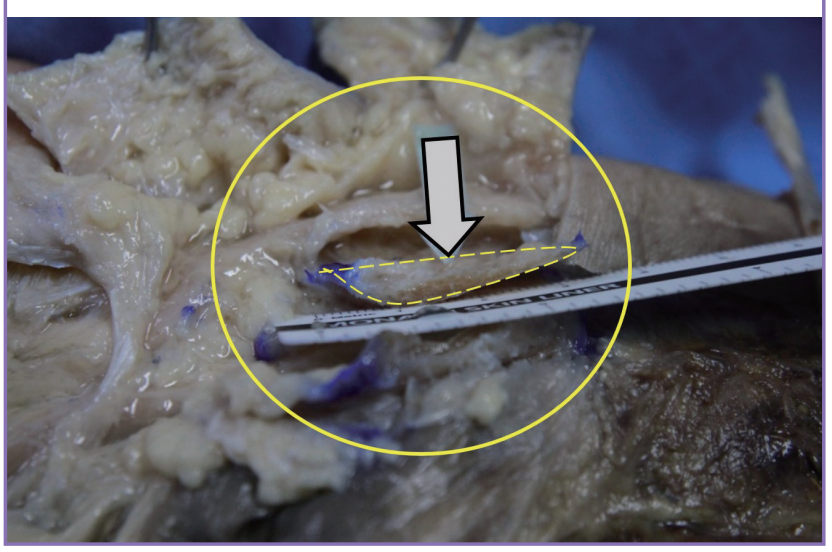

structures. The average length between the distal wrist crease and the distal part of the TCL was $35.302 .59 \mathrm{~mm}$ (range, 32-36 $\mathrm{mm}$ ), the average thickness of the thickest region was $4.00 \mathrm{~mm} \pm$ $0.57 \mathrm{~mm}$, and the average distance from the distal TCL to the superficial palmar arch was $9.50 \pm 2.13 \mathrm{~mm}$ (Table 1 ). The course of the superficial palmar arch was distal to the TCL and in close proximity to the ulnar side of the third web towards the transverse distal to the TCL. The average distance from the thickest part of the TCL to the distal wrist crease was $25.00 \pm 0.71 \mathrm{~mm}$ (Table 1). The proximal end of the TCL was connected to the flexor retinaculum with the thin fascia (Figs. 2, 3). Fig. 4 shows the schematic representation of the relationship between the TCL and the adjacent structures. The thickest part $(4 \mathrm{~mm})$ of the TCL was measured to be $25.00 \mathrm{~mm}(25.00 \pm 0.71 \mathrm{~mm})$ distal from the distal wrist crease.
Fig. 4. Schematic presentation of the TCL and adjacent structure

The thickest part $(4 \mathrm{~mm}$ ) of the TCL was measured $25 \mathrm{~mm}$ distal from the DWC. Sufficient resection of the TCL up to $25-35 \mathrm{~mm}$ distal from the distal wrist crease can ensure reduced compression in this region. However, special care should be taken to not exceed the limit, because a resection with a pair of scissors exceeding $35 \mathrm{~mm}$ can pass the fat pad and damage the SPA. SPA, superficial palmar arch; TCL, transverse carpal ligament; DWC, distal wrist crease.

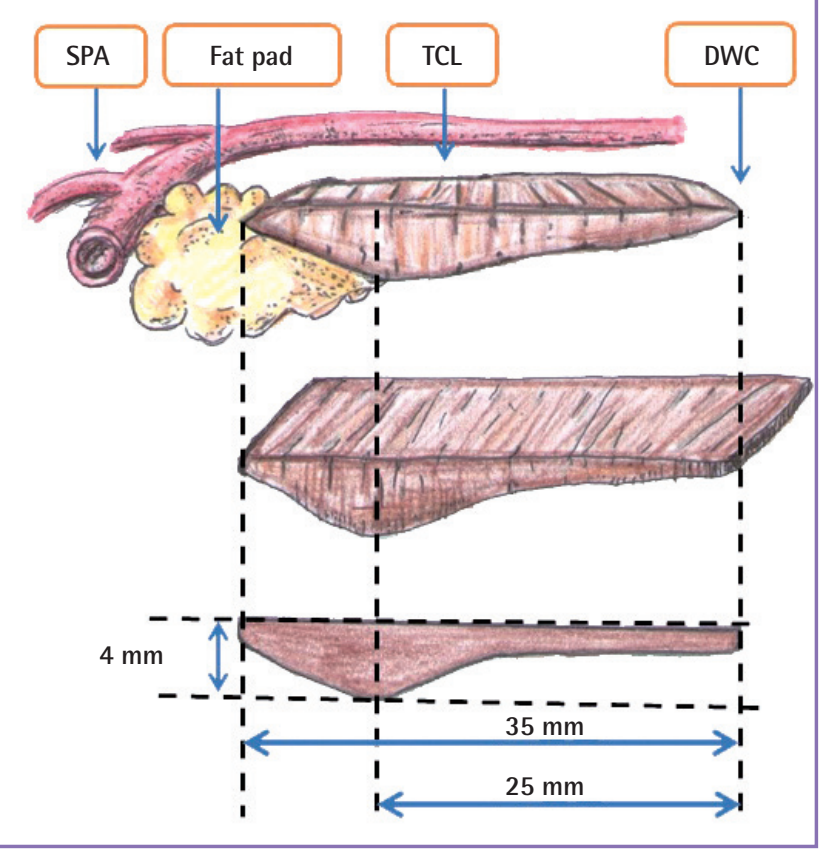

\section{Clinical study}

Table 2 shows detailed information for the patient group. Thirteen patients underwent fasciotomy with a minimally invasive incision of the distal wrist crease. One case in one patient (case 11) with symptoms lasting more than 20 years yielded a "bad" result (less than 50\% postoperative recovery of hand functions). In this case, the two-point discrimination test yielded an aggravated sensation. Except this case, the 12 other cases proved successful by yielding satisfactory or very satisfactory results (Table 2 ). Of the 13 patients, decreased sensation was observed in 2 cases $(15.3 \%)$ and no change in sensation was observed in the remaining 11 cases $(84.7 \%)$. There were two cases $(15.3 \%)$ of scar discomfort (hypertrophic scar and pillar pain) and no scar discomfort in the other 11 cases (84.7\%) (Table 2).

\section{DISCUSSION}

CTS is the most frequently encountered peripheral compressive neuropathy and is known to be curable with surgical intervention in which the transverse ligament of the carpal tunnel is released by resection. Of a wide variety of therapeutic interventions, the current standard treatment is the open release of the 
Table 2. Patient details

\begin{tabular}{|c|c|c|c|c|c|}
\hline Case & Age/Sex & Underlying disease & Outcome & Change in sensation & Scar discomfort \\
\hline 1 & 70/Female & DM & Excellent & No & No \\
\hline 2 & 57/Female & DM, HTN & Excellent & No & No \\
\hline 3 & 62/Male & Pyelonephritis & Excellent & No & No \\
\hline 4 & 58/Female & DM & Good & Decreased & Hypertrophic scar \\
\hline 5 & 58/Male & No & Excellent & No & No \\
\hline 6 & 62/Female & DM & Good & No & No \\
\hline 7 & 60/Male & Hepatitis & Excellent & No & No \\
\hline 8 & 48/Female & CRF, DM CKD, angina & Good & No & No \\
\hline 9 & 54/Female & No & Excellent & No & No \\
\hline 10 & 53/Female & Osteoporosis & Excellent & No & No \\
\hline 11 & 51/Female & HTN, angina & Poor & Decreased & Pillar pain \\
\hline 12 & 40/Female & No & Excellent & No & No \\
\hline 13 & 47/Female & HTN & Excellent & No & No \\
\hline
\end{tabular}

TCL, which involves total resection of the subcutaneous tissues, palmar fascia, transverse fibers, mid-palmar fascia, distal flexor retinaculum, forearm fascia, and TCL [10]. Common postoperative complaints include tenderness at the surgical site, a tingling sensation, burning pain, deep pain, pillar pain, dysesthesia, and hypesthesia, collectively called scar discomfort. The incidence of scar discomfort reported by different researchers varies between 19\% and 61\%; however, its exact cause is yet to be explored and understood. Some authors attribute scar discomfort to the consequences of neuroma formation owing to palmar cutaneous branch damage. Some anatomical studies have presented new skin incision sites based on extensive investigation of the palmar skin and neural distribution, but palmar cutaneous branch damage cannot be completely avoided in open surgery [11]. Although the endoscopic approach has contributed to rapid progress in the prevention of scar discomfort, the symptom of CTS is prone to recurrence. Moreover, with cadaver autopsies revealing incomplete resection of the TCL, the currently recognized standard treatment is total resection of the TCL [12]. However, such conventional surgical methods require a wide opening of the skin and inner structures, thus placing patients at a high risk of postoperative scar formation and pain. Moreover, the postoperative increase in the space inside the carpal tunnel and scar formation triggers dislocation of the tendon during flexor contraction, resulting in postoperative complications such as anterior draw of the flexors, pain at the surgical site, and loss of grip.

Cellocco et al. [13] and Hamed and Harfoushi [14] reported on mini-open procedures for carpal tunnel release. However, compared to previous studies, in this study, different confirmation methods were used for the complete resection of the TCL. The present research shows through an anatomical study that TCL fasciotomy with a minimally invasive incision of the distal wrist crease is a safe surgical method that does not cause damage to the surrounding structure. Furthermore, this research emphasizes that ensuring the surgical release of the thickest part of the TCL is extremely important.

Among TCL-related anatomical studies, Rotman and Donovan [15] reported that the average length of the TCL was 24-36 $\mathrm{mm}$ with thin proximal $(6-20 \mathrm{~mm})$ and distal $(6-10 \mathrm{~mm}) \mathrm{sec}-$ tions and that the middle-to-distal section was the thickest part. Further, in these previous studies, the researchers identified the region $10-40 \mathrm{~mm}$ from the distal wrist crease as that exposed to the severest compression of the median nerve [16] and the region 5-15 mm distal to the distal part of the TCL as that exposed to the greatest compression of the TCL [17].

Lee et al. [12] performed a study with 24 cadavers and noted that the TCL length ranged from 19 to $55 \mathrm{~mm}$ (average, $29 \mathrm{~mm}$ ). Collectively, these results indicate that the region proximal to the TCL may be the most important target for resection in the treatment of CTS. However, these studies included Caucasians, and the present study, which included only Koreans, determined that the thickest part was the region $25 \mathrm{~mm}$ distal from the most proximal part of the TCL. Therefore, the successful release of compression can only be achieved by precise resection up to this region, which should be exposed to the greatest compression.

In this study, the TCL length differed among the patients, presumably due to different wrist and hand sizes. Sunil et al. [18] studied the relationships between hand size and body height with 150 subjects and reported that body height is proportional to the length from the proximal wrist crease to the tip of the middle finger. Given the potential of this relationship between the cadaver body height and TCL length, the relationship will have to be investigated in future research. However, considering the age group of the patients that typically develop CTS (middle- 
aged people) and the average age in relation to the average body height of Korean adults of both sexes, the potential variation is considered negligible.

During the present study, we determined that the TCL begins anatomically from the distal wrist crease and measures an average of $35 \mathrm{~mm}$. Therefore, we believe that an accurate preoperative drawing of the distal part of the TCL can prevent damage to the superficial palmar arch resulting from excessive resection of the distal region with a pair of scissors. Moreover, special care should be taken to not exceed the limit, because a resection with a pair of scissors exceeding $35 \mathrm{~mm}$ can pass the fat pad and damage the superficial palmar arch (Fig. 4). We also confirmed that if the resection scissors follow the longitudinal line drawn from the apex of the third web space to the radial side, superficial palmar arch damage can be substantially reduced, because the superficial palmar arch is generally approximately $10 \mathrm{~mm}$ from the distal part of the TCL. Because the TCL is the thickest $(4 \mathrm{~mm})$ at $25 \mathrm{~mm}$, on average, from the distal wrist crease, we also determined that it is of vital importance that this be completely released. This is the crucial part that causes a tingling feeling when compression is applied on the median nerve because it is the thickest region in the TCL and the medial nerve branches distal to this region. Sufficient resection of the TCL up to $25-35 \mathrm{~mm}$ distal from the distal wrist crease can ensure reduced compression in this region. When we applied these anatomical findings in a clinical setting, we found that a minimally invasive incision of the distal wrist crease alone was sufficient for successful surgery, and the time to recovery was much shorter because the wrist crease region was thinner than the palmar region. Moreover, given that patients with comorbidities such as diabetes mellitus and the nephrotic syndrome can experience delayed wound recovery, other surgical methods involving a palmar incision can increase the risk of complications. Therefore, particularly with such patients, a minimally invasive incision of the distal wrist crease can be considered the best method.

This study has several limitations. First, there were considerable intersubject differences in the distance between the distal part of the TCL and the superficial palmar arch. Second, the cadavers were not fresh, and the number of cadavers was small. Third, a learning curve was involved because blind fasciotomy was required when there was insufficient visibility. Fourth, the thickness of the palmar fat pad varied among individuals. To further our research, we need a large number of fresh cadavers for dissection, and extensive tests and follow-up are necessary to monitor damage to the palmar branch of the median nerve.

In conclusion, TCL fasciotomy with a minimally invasive incision of the distal wrist crease is a method that involves a steep learning curve; however, it leaves only a minimal scar that is less visible in the carpal crease compared with other surgical methods. Moreover, it is considered an effective approach because of its minimally invasive approach that results in a shorter recovery time and reduced incidence of complications.

\section{REFERENCES}

1. Paget J. Lectures on surgical pathology. 2nd ed. Philadelphia: Lindsay \& Blakiston; 1854.

2. Pfeffer GB, Gelberman RH, Boyes JH, et al. The history of carpal tunnel syndrome. J Hand Surg Br 1988;13:28-34.

3. Learmonth JR. The principle of decompression in the treatment of certain diseases of peripheral nerves. Surg Clin North Am 1933;13:905-13.

4. Atroshi I, Gummesson C, Johnsson R, et al. Prevalence of carpal tunnel syndrome in a general population. JAMA 1999; 282:153-8.

5. Graham B, Regehr G, Naglie G, et al. Development and validation of diagnostic criteria for carpal tunnel syndrome. J Hand Surg Am 2006;31:919-24.

6. Kim DH. Carpal tunnel syndrome: open carpal tunnel release. J Korean Neurotraumatol Soc 2008;4:1-7.

7. Tinel J. Le signe du fourmillement dans les lesions des nerfs peripheriques. Presse Med 1915;23: 388-9.

8. Phalen GS. The carpal tunnel syndrome: seventeen years' experience in diagnosis and treatment of 654 hands. J Bone Joint Surg Am 1966;48:211-28.

9. Bezuhly M. Nerve entrapment syndrome. In: Neligan PC, editor. Plastic surgery. 3rd ed. Philadelphia: Elsevier Saunders; 2013. p.507.

10. Okutsu I, Hamanaka I, Tanabe T, et al. Complete endoscopic carpal canal decompression. Am J Orthop (Belle Mead NJ) 1996;25:365-8.

11. Watchmaker GP, Weber D, Mackinnon SE. Avoidance of transection of the palmar cutaneous branch of the median nerve in carpal tunnel release. J Hand Surg Am 1996;21:64450.

12. Lee DH, Masear VR, Meyer RD, et al. Endoscopic carpal tunnel release: a cadaveric study. J Hand Surg Am 1992;17: 1003-8.

13. Cellocco P, Rossi C, Bizzarri F, et al. Mini-open blind procedure versus limited open technique for carpal tunnel release: a 30-month follow-up study.J Hand Surg Am 2005;30:493-9.

14. Hamed SA, Harfoushi FZ. Carpal tunnel release via miniopen wrist crease incision: procedure and results of four years clinical experience. PakJ Med Sci 2006;22:367-72.

15. Rotman MB, Donovan JP. Practical anatomy of the carpal tunnel. Hand Clin 2002;18:219-30. 
16. Kang YK, Kim DH, Lee SH, et al. Tenelectrodes: a new stimulator for inching technique in the diagnosis of carpal tunnel syndrome. Yonsei Med J 2003;44:479-84.

17. Ikeda K, Osamura N, Tomita K. Segmental carpal canal pres- sure in patients with carpal tunnel syndrome. J Hand Surg Am 2006;31:925-9.

18. Sunil, Dikshit PC, Aggrawal A, et al. Estimation of stature from hand length. J Indian Forensic Sci 2005;27:219-21. 